\title{
Experimental Investigation of Steady and Unsteady Flow Field Upstream and Downstream of an Automotive Torque Converter Pump
}

\author{
B.V. MARATHE and B. LAKSHMINARAYANA* \\ Department of Aerospace Engineering, The Pennsylvania State University, University Park, PA 16802, USA
}

(Received 18 May 1997; In final form 15 May 1998)

\begin{abstract}
The objective of this investigation is to understand the steady and the unsteady flow field at the exit of an automotive torque converter pump with a view towards improving its performance. The measurements were conducted in a stationary frame of reference using a high frequency response five-hole probe and the data were processed to derive the flow properties in the relative (pump) frame of reference. The experimental data were processed at three different operating conditions: maximum efficiency point, design point and near-stall point. The unsteady values of flow properties (pressure, velocity and flow angles) were divided into five components: mean, periodic, blade aperiodic, revolution aperiodic and unresolved components.

The velocity profiles indicate zones of separation near the core region at speed ratio (SR) 0.8. This zone is transported to the shell region at SR 0.065 due to the presence of a strong secondary vortex. The secondary vortex (weak) for the SR 0.8 rotates anti-clockwise, and is located only near core-wake region. The secondary vortex (strong) at SR 0.065 rotates clockwise, and encompasses the entire passage. The unsteady flow data show that unresolved and periodic components dominate the unsteadiness at the pump exit. The overall aperiodicity is negligible and is dominated by the blade aperiodic component.
\end{abstract}

Keywords: Torque converter, Pump, Five-hole probe, Measurements, Steady and unsteady

\section{INTRODUCTION}

The torque converter flow field is very complex. It is three dimensional, viscous and unsteady due to the close coupling between rotor and stator elements. Additional complexities arise due to differential rotor speeds of the pump and the turbine. The unsteadiness is generated due to potential and viscous interactions at the rotor-rotor and the rotor-stator interfaces. The turbine and the pump passages are narrow, long and are dominated by viscous effects, separation and secondary flow.

* Corresponding author. 153 Hammond Bldgs, University Park, PA 16802, USA. Tel.: (814) 865-5551. Fax: (814) 865-7092. E-mail: bllaer $(a)$ engr.psu.edu. 
The nature and magnitude of the unsteadiness varies with several parameters including spacing between two blade rows, number of blades in both the rotor and the stator, thickness of the blade trailing edge, location of flow separation, secondary flows and other geometrical and operating parameters.

There have been several attempts made in recent years to investigate the torque converter flow field (e.g. Brun et al., 1996; Marathe et al., 1997; Gruver et al., 1996; By et al., 1995; Browarzik and Grahl, 1992). A brief review of this is given in Backström and Lakshminarayana (1996). However, none of the prior investigators report detailed steady and unsteady pressure and velocity at the exit of the pump. The thrust of this paper is to understand the steady and the unsteady flow field at the exit of the pump at maximum efficiency and stall conditions. The flow investigation by Brun et al. (1996) and By et al. (1995) has shown that the flow field inside the pump passages is three dimensional, unsteady and complex with large regions of flow separation and strong secondary flows. Three phenomena play significant role in the flow structure: (1) Simultaneous flow turning in tangential as well as azimuthal direction generates strong secondary flow pattern inside the blade passage. (2) The temporal and spacial variation in the incidence angle generates unsteady flow separation on the core and the suction surface. (3) The upstream and downstream pressure variations combined with upstream secondary flow generates highly unsteady and complex flow patterns causing high levels of unsteadiness and turbulence.

The objective of this investigation is to understand the steady and the unsteady flow structure at inlet and exit of the pump (including pressure and velocity) and analyze the data to evaluate the performance of the pump. The experimental work is carried out using a miniature fast-response fivehole probe at the exit of the pump. The stator exit flow field was surveyed using a conventional fivehole probe.

Since the flow field under consideration is the pump inlet and exit flow field, the properties are presented only in the relative (pump) frame of reference. However, the radial distributions of the mass averaged quantities are presented in both the relative and the absolute frame of reference to compare the radial variation of the flow properties at the exit of each component. The radial distributions of RMS unsteadiness are presented only in the absolute reference frame.

\section{FACILITY AND INSTRUMENTATION}

The torque converter test facility has five components: driver and absorption dynamometer, test section, oil system and control system. The details of the facility are given in Marathe et al. (1997). The number of blades on the pump, turbine and stator are 27, 29 and 19, respectively. An oil system is used to keep the oil pressure and temperature constant at approximately $14 \mathrm{psi}$ and $60^{\circ} \mathrm{C}$, respectively. The oil used is a non-conductive, non-corrosive oil with a density of $857 \mathrm{~kg} / \mathrm{m}^{3}$ at $60^{\circ} \mathrm{C}$. The performance of a torque converter is expressed in terms of torque ratio (TR) and speed ratio (SR), defined in the nomenclature.

Tests were conducted at $\mathrm{SR}=0.0,0.2,0.4,0.6$ and 0.8 to simulate all the conditions the torque converter experiences in actual automatic transmission. The pump rpm at these speed ratios are 1050, 1070, 1090, 1160 and 1300, respectively. The design speed ratio of the torque converter is 0.6 . It has a maximum efficiency at $\mathrm{SR}=0.8$ and minimum efficiency at $\mathrm{SR}=0.0$. The data acquired at the exit of the pump at the design, maximum efficiency and minimum efficiency speed ratios are presented and interpreted in this paper. Measurements are carried out at five radial positions and one tangential position at the pump exit.

A miniature fast-response five-hole probe (1.67 $\mathrm{mm}$ tip diameter) was designed and fabricated to facilitate the measurements of the flow properties upstream and downstream of each of the blade rows. Details of the five-hole probe have been presented by Marathe et al. (1997). Five miniature pressure transducers were mounted inside the five-hole probe. This allowed extremely 
small cavity volume between the probe tip and the pressure transducer. The cavity between the probe tip and the pressure transducer was filled with oil to improve the response time of the probe. The tests performed in a hydro-acoustic chamber indicate a frequency response of approximately $8 \mathrm{kHz}$. This frequency response was considered sufficient since the maximum blade passing frequency is $585 \mathrm{~Hz}$. A pressure tap was installed on the stator shell surface (hub) for measuring the hub static pressure. This static pressure was used for normalizing the stagnation and static pressures measured by the five-hole probe upstream and downstream of the pump. The amplified signal from the pressure transducer was acquired and recorded by the DAS-50 data acquisition system. A signal from the shaft encoder, mounted on the pump shaft, was used as clocking frequency to maintain the data acquisition rate precisely at 50 times the blade passing frequency of the pump.

A thermocouple was installed on the hub of the stator for accurate reference temperature. The probe calibration has an accuracy of $\pm 1^{\circ}$ in angle measurement. The pressure measurements are obtained to an accuracy of \pm 0.01 psi. The transducer drift introduces a maximum error of $0.5 \%$. This results in a maximum $1-2 \%$ error in the velocities and $0.5 \%$ error in the static and total pressures. Corrections to the probe due to the blockage and the pressure gradient are incorporated into the data processing. The maximum cumulative error in the pressure measurements is estimated to be $2-3 \%$.

\section{SIGNAL PROCESSING AND AVERAGING TECHNIQUE}

The unsteady pressure data from the five-hole probe were processed to obtain the instantaneous values of $P_{0}, P_{\mathrm{S}}, V, \alpha$ and $\beta$. The data were processed digitally and also through a spectrum analyzer to determine the dominant frequencies. The dominant frequency downstream of pump was found to be that due to the pump blade passing frequency. It is beyond the scope of this paper to discuss the details of the spectrum analysis.

The signal from the pressure transducer was decomposed to deterministic and unresolved components. Since, the deterministic signal is generated by the relative motion between the stator and the rotor blade passages and the blade-to-blade variation of properties, it repeats over every revolution in conventional turbomachinery. However, for the torque converter, the pump and the turbine rotors have different rotational speeds. As a result, flow conditions do not repeat over one revolution of any rotor. Hence, it was necessary to find the period over which the deterministic part of the signal repeats itself. At $\mathrm{SR}=0.8$ the pump and the turbine shaft speeds (rps) are in $5: 4$ proportion. Hence, five revolutions of the pump and four revolutions of the turbine are completed in the identical time period. Time required to complete five revolutions of the pump will be referred to as "cycle" in this paper. All deterministic signals are considered repeatable over every cycle. Hence, ensemble averaging technique was employed over one cycle instead of one revolution. A detailed explanation of this method is provided in Marathe et al. (1997).

\section{PUMP UPSTREAM FLOW IN RELATIVE REFERENCE FRAME}

Relative stagnation pressure coefficient, $\left(C_{P 0}\right)_{\mathrm{REL}}$, distribution, shown in Fig. 1, at the pump inlet for SR 0.8 reveals higher values near the core and lower values near the shell. A thick diffused stator wake is observed near the suction side. The variation of the stagnation pressure coefficient is small in the tangential direction and large in the radial direction.

It should be remarked here that the pump inlet flow is dominated by stator wakes and end-wall flows. The static pressure is higher and the absolute velocity is lower at the core, hence the reason for higher values of $\left(C_{P 0}\right)_{\mathrm{REL}}$. The measurements at the leading edge of the pump (Brun et al., 1996) 


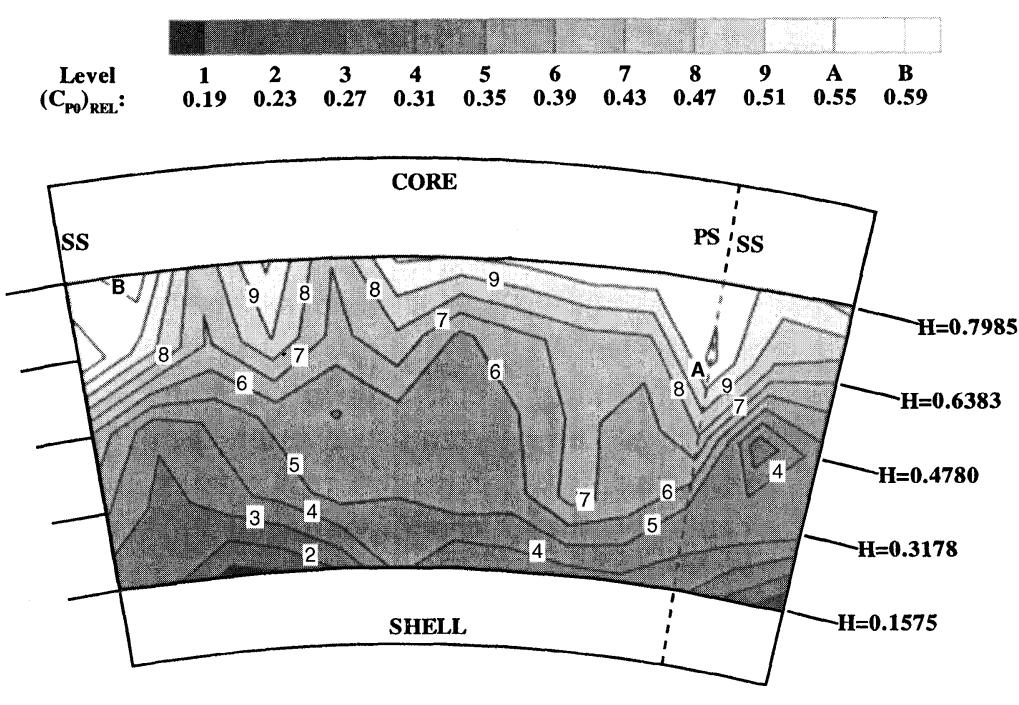

$\underline{\text { SR } 0.8}$

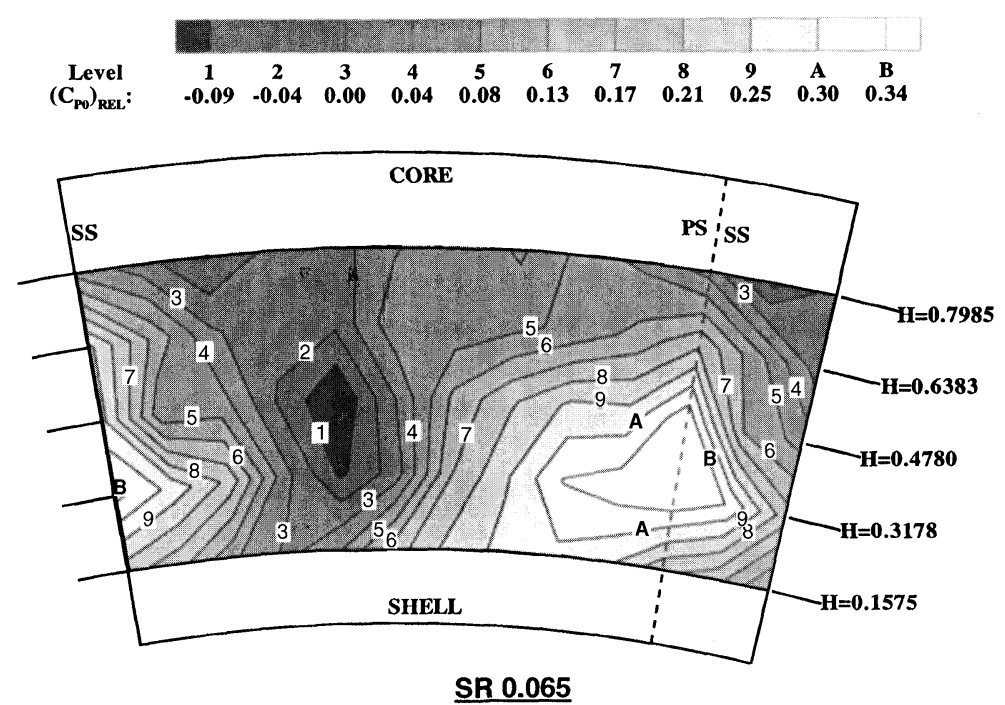

FIGURE 1 Coefficient of relative stagnation pressure $\left(C_{P 0}\right)_{\text {REL }}$ at SRs 0.8 and 0.065 at pump inlet.

indicate that the absolute flow near the core is separated and the relative flow is nearly tangential resulting in high incidence angles to the pump rotor. The shell region, on the other hand, has higher absolute flow and lower relative flow velocity resulting in moderate incidence into the pump rotor. The concave curvature that exists at inlet near the shell from the stator exit to the pump inlet should enhance this trend (higher absolute and lower relative velocities).

The stagnation pressure coefficient at SR 0.065 (Fig. 1) shows a large region of low $\left(C_{P 0}\right)_{\mathrm{REL}}$ which indicates thick wake and possible flow separation near the suction side of stator. This zone is observed in both the absolute and the relative reference frame. The low momentum region in the 
absolute reference frame becomes high momentum region in the relative reference frame only inside and near the suction side of the wake/separation region. However this region also shows low values of $\left(C_{P 0}\right)_{\mathrm{REL}}$ due to low $C_{P \mathrm{~S}}$ values in that region. In all other areas, the distribution of stagnation pressure coefficient remains similar both in relative and absolute reference frames (Marathe,.1998). This is attributed to the high flow overturning near the suction side of the wake/separation zone. The velocity triangles are highly skewed and the relative velocity vectors remain in the same direction as the absolute velocity vectors. Most of the variation in the stagnation pressure coefficient is in the tangential direction due to the presence of thick wake which stretches from shell to core near the suction side. This generates significant amount of periodic unsteadiness in the pump flow field at that speed ratio. It is evident that $\left(C_{P 0}\right)_{\mathrm{REL}}$ values show higher tangential variation as speed ratio decreases from 0.8 to 0.065 . The upstream tangential variation of $\left(C_{P 0}\right)_{\mathrm{REL}}$ variation has a significant influence on the regions of unsteady flow separation and secondary flow pattern inside the pump blade passages. Therefore contribution to the periodic unsteadiness in overall unsteadiness increases as the speed ratio decreases. However, at lower speed ratios the random unsteadiness decreases significantly due to lower pressure rise in the pump and higher mass flows.

Normalized relative total velocity contours (Fig. 2) at SR 0.8 show low velocity region near the suction surface core and the high velocity region near the pressure surface shell. The variation in the velocity indicates a thin diffused wake at the pump inlet. The low momentum regions in the absolute reference frame become high momentum regions in the relative reference frame and vice versa. The overall variation is small $(16 \%$ of the reference velocity). Most of the variation occurs between mid-span and core region near the wake. The stator wakes are relatively thin away from the core and shell, with high free-stream absolute velocity (low relative velocity) occurring near the suction surface, an inviscid effect. The radial variation in the relative velocity is not large.

At SR 0.065, the normalized relative total velocity contours (Fig. 2) show a significant variation in the magnitude from the pressure to the suction surface. The tangential variation of the velocity is higher than the radial variation. Since the velocity triangles are skewed (i.e. the relative and absolute total velocity vectors are in same direction) the wake in the absolute reference frame remains wake in the relative reference frame and high momentum regions in the absolute reference frame remain high momentum regions in relative reference frames. The thick wake/separation region is clearly evident near the suction side. This variation in the velocity generates high variation of the incidence angles and thus high levels of unsteadiness inside the pump.

The relative yaw angles at the pump inlet, at SR 0.8 (Fig. 3) reveal a significant variation (approximately $30^{\circ}$ ) upstream of the pump. However, most of the variation is in the radial direction. The tangential variation is only $10^{\circ}$ across the wake. The inviscid core flow shows less than $5^{\circ}$ variation in the tangential direction. The shell region shows flow angles of approximately $-32^{\circ}$. Since the blade angle of the pump is $-35^{\circ}$, the pump incidence angles near the shell are small. However, near the core the flow angles are approximately $62^{\circ}$. This generates extremely high positive incidence angles near the core. This causes a flow separation and reattachment near the leading edge suction surface of the pump as observed by By et al. (1995). Such large angles near the core result in leading edge separation. Since the flow encounters convex surface from stator trailing edge to pump leading edge along the core, the absolute flow is decelerated attenuating the high incidence effect observed.

At SR 0.065, the flow angle distribution (Fig. 3) is significantly different than at SR 0.8 . A significant variation in the flow angles occurs in the tangential as well as the radial direction due to the thick wake/ separation region observed. The flow angles vary by about $75^{\circ}$ in the tangential direction and about 

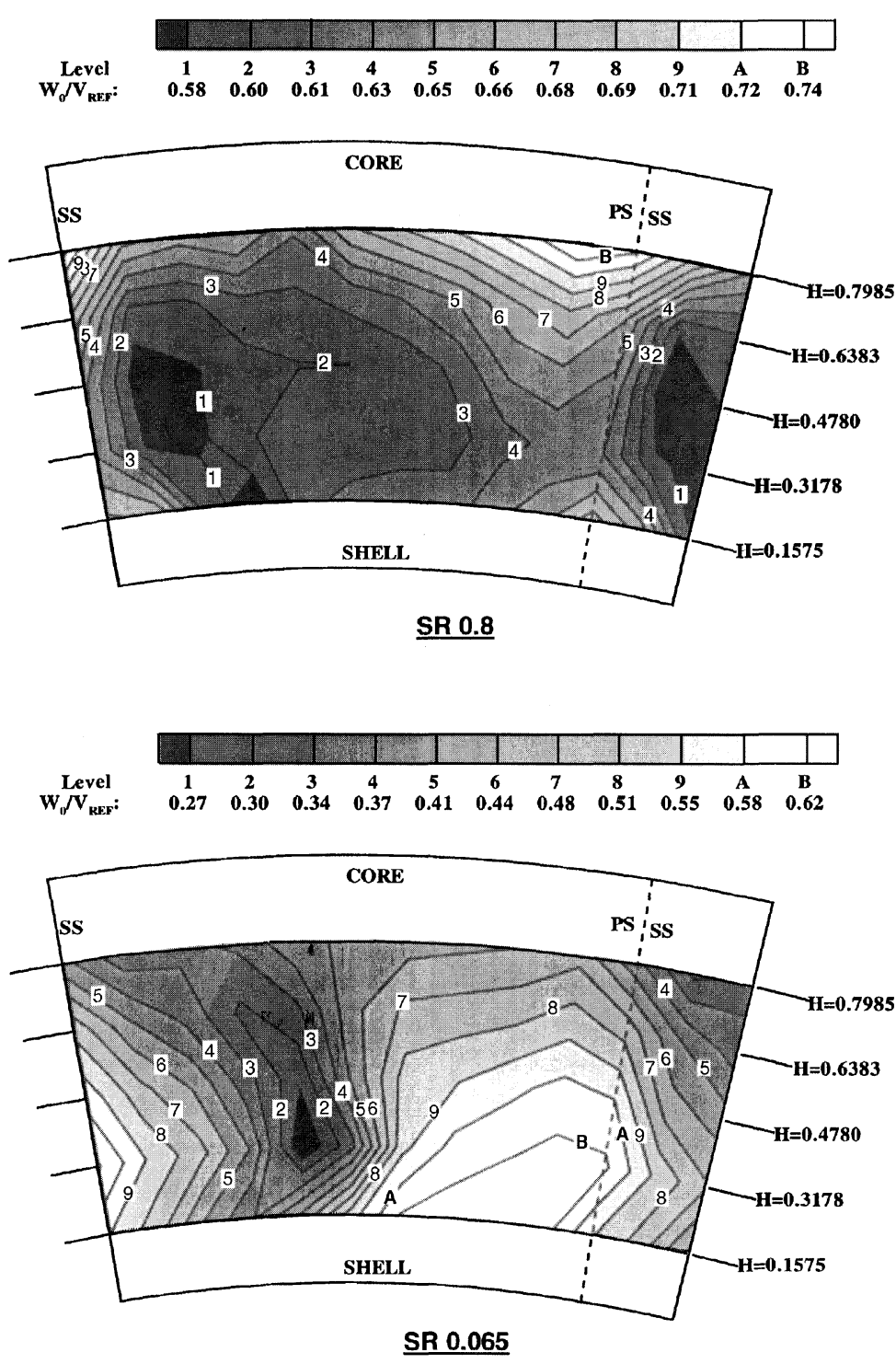

FIGURE 2 Contours of normalized relative total velocity at SRs 0.8 and 0.065 at pump inlet.

$45^{\circ}$ in the radial direction. The flow angles are close to the pump blade angles only in the wake/ separation zone near the mid-span. In the wake, the incidence angles vary from $-50^{\circ}$ near the shell to about $+10^{\circ}$ near the mid-span to about $-40^{\circ}$ near the core. Whereas, in the inviscid core region of the flow the incidence angles vary from $-60^{\circ}$ near shell to $-50^{\circ}$ near mid-span to $-45^{\circ}$ near the core.
This generates highly pulsating flow inside the pump near the mid-span, and flow separation near the pressure side of the pump blades near the shell and core. The location and size of separated flow regions inside the pump passage is influenced by the upstream flow angles. The variation in the upstream flow angles also affect the nature of secondary flow inside the pump passage. 

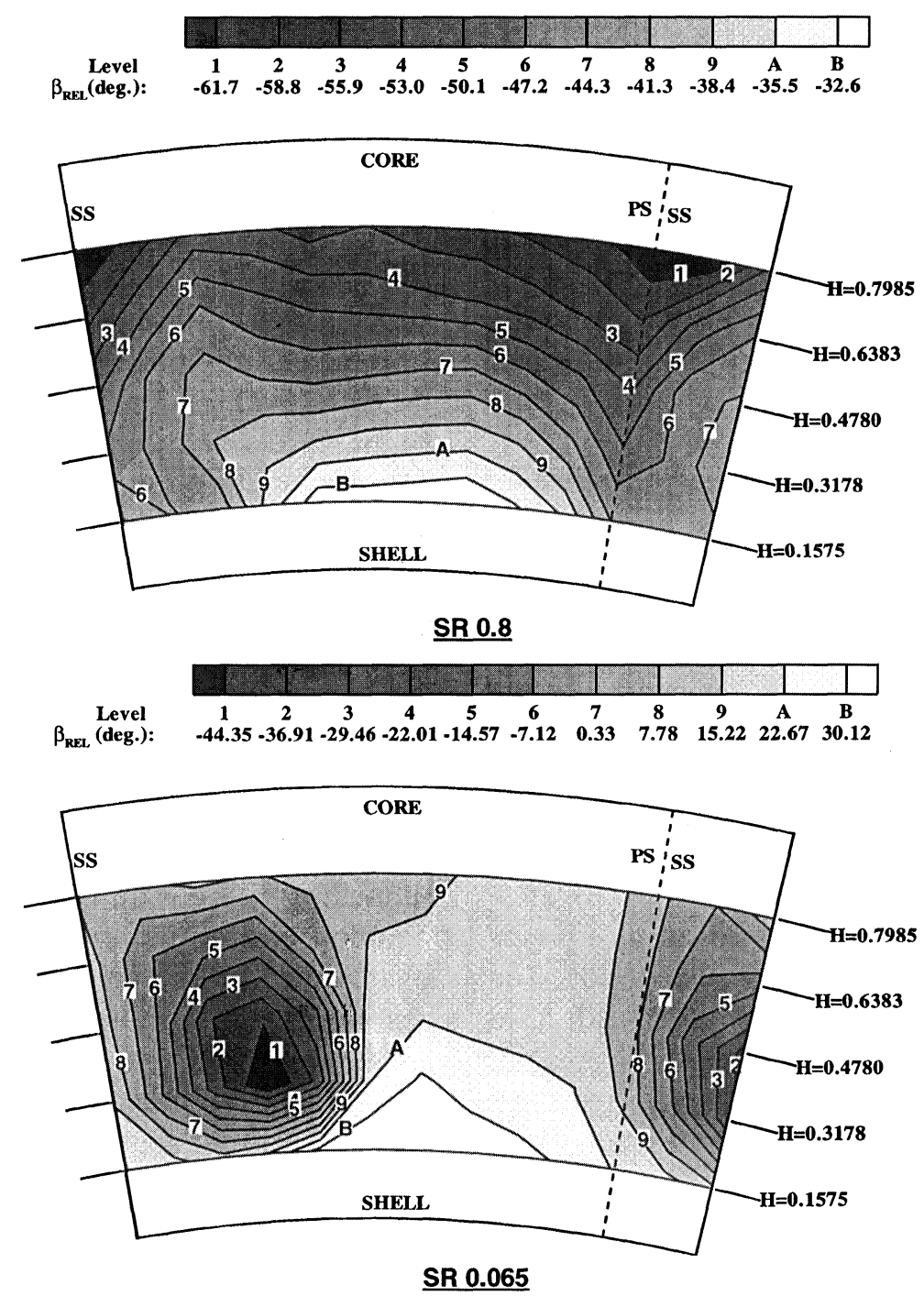

FIGURE 3 Contours of relative yaw angles at SRs 0.8 and 0.065 at pump inlet.

\section{PUMP EXIT FLOW IN \\ RELATIVE REFERENCE FRAME}

The flow properties at the pump exit are presented in this section. All the data presented in this section are steady state ensemble averaged data in the pump reference frame. The unsteady data in absolute reference frame are presented in the next section.
The ensemble averaged total velocity in consecutive blade passages at SR 0.8 (not presented) shows that the flow is nearly periodic with aperiodicity in certain regions. The fact is apparent from the low levels of aperiodic fluctuations presented later in this paper. The relative positions of low and high momentum regions are periodic. Two phenomena cause aperiodicity in the pump flow field: (1) The relative position of the turbine and stator blade 
passages. This causes tangential non-uniformity in the pump flow passages. However, for present investigation the aperiodicity measured is the effect of only turbine blade position (and not stator blade position) as the five-hole probe's relative position with the stator blade was maintained identical for all the measurements. (2) The temporally aperiodic flow phenomenon like blade-to-blade variation in the wake profile, unsteady flow separation and reattachment in flow passages, etc.

\section{Stagnation and Static Pressure Coefficient at Pump Exit}

The distribution of relative stagnation pressure coefficients $\left(C_{P 0}\right)_{\mathrm{REL}}$, at speed ratios 0.8 and 0.065 is shown in Fig. 4. The relative stagnation pressure near the shell (about $\frac{1}{3}$ of span) is nearly uniform in the tangential direction, with large gradients in the radial direction. The pump rotor wake has decayed substantially in this region. The region $H=0.37$ to $H=1.0$ (core) also has nearly uniform relative pressure coefficient, but their values are much lower than those encountered near the shell region. The variation of the dynamic pressure does not have significant influence on the total relative pressure variation as the total relative velocity is small at the pump exit. Wake from the pump blade is completely dissipated before it reaches the measurement plane. This can be attributed to the wake decay caused by large distance along the absolute streamwise direction from trailing edge of the pump to the measuring plane. Although, the axial distance between the trailing edge of the pump blade and the probe tip is very small (approximately $4 \mathrm{~mm}$ ), the streamwise distance is large (approximately $30-40 \mathrm{~mm}$ ) due to high flow angles $\left(80-90^{\circ}\right)$ in the absolute reference frame. Also, the wake dissipation is enhanced near the core and the shell due to the flow deceleration, caused by the presence of a stationary hub and a turbine shell with slow speed of rotation, at the measurement location. A low stagnation pressure region is observed near the shell core region. The reasons for the lower stagnation pressure near the core are as follows: (1) The flow separation near the core surface inside the pump passage as observed by Brun et al. (1996). This causes the transportation of high momentum flow region towards the shell. (2) This momentum transport is enhanced further due to leakage of flow at the shell near the measurement plane. This is confirmed by the high radial outward velocities near the shell as discussed later in this chapter. (3) Inlet flow distribution shows lower mass flow near the core. (4) The pump flow enters the turbine shell before it reaches the measurement plane. The difference in the rotational speed of the pump and the turbine causes considerable deceleration of the flow. This generates blockage in the radially outward flow and high values of static pressure near the shell. Since, this phenomenon occurs only near the shell, only the measurement radius closest to the shell show high values of static pressure. All these reasons contribute in generating significantly high dynamic and static pressure near the shell than near the core. The static pressure near the core (presented later) is low due to low values of diffusion inside the pump passage. Therefore significantly lower values of total pressure are observed near the core for all speed ratios. It should also be observed that the distribution of the relative total pressure coefficient is influenced mainly by the distribution of the static pressure coefficient as the relative dynamic head is insignificant at pump exit for SR 0.8 (Fig. 5).

For SR 0.065 , the distribution of relative total pressure coefficient (Fig. 4) is similar to that observed for SR 0.8. However, the overall magnitude of the coefficient is small because of low $C_{P 0}$ rise in the pump. Therefore, magnitudes of the tangential variation for $\left(C_{P 0}\right)_{\mathrm{REL}}$ are negligible. The radial variation of the relative stagnation pressure is significant only near the shell and is influenced by the radial variation of the static pressure. For SR 0.065 the magnitudes of exit total pressure are small due to low rotor speed and high mass flow. Lower static pressure near the core and higher static pressure near the shell is caused by lower diffusion of velocities near the core and higher diffusion near the shell. This is attributed to variation in the area 

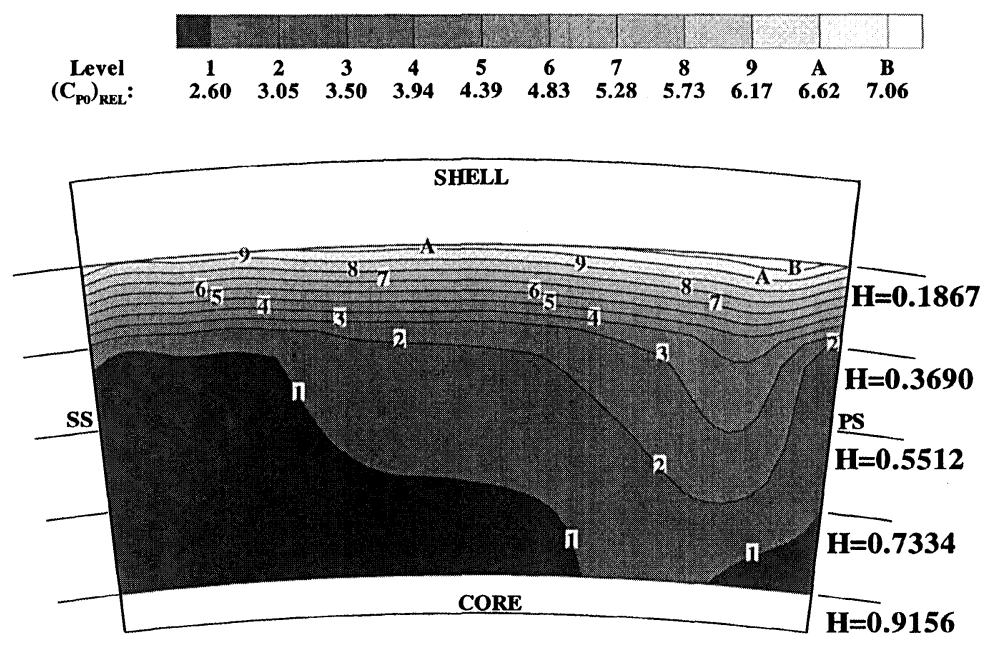

$\underline{\text { SR } 0.8}$

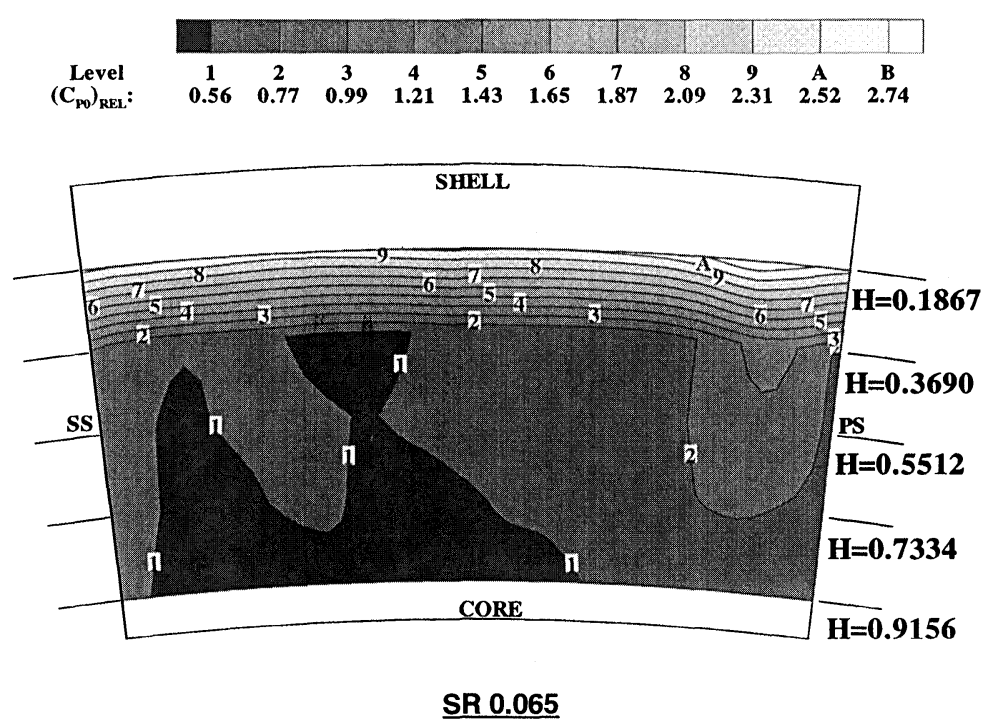

FIGURE 4 Coefficient of relative stagnation pressure at SRs 0.8 and 0.065 at pump exit.

ratio inside the pump passage from core to shell. Another reason for lower static pressure near the core is higher losses and smaller loading near the core region.

The radial distribution of passage averaged relative and absolute total pressure at SR 0.8 (Fig. 5) shows that the relative total pressure decreases sharply from shell to $H=0.3690$ and decreases smoothly from $H=0.3690$ to core for SR 0.8 . For SR 0.065 , the radial distribution of passage averaged absolute stagnation pressure (Fig. 5) shows a significant drop from shell to $H=0.3690$ and then remains nearly uniform in the rest of the passage with a slight increase in stagnation pressure from $H=0.3690$ to core. This can be attributed to a slight increase in the dynamic head from core to shell. 


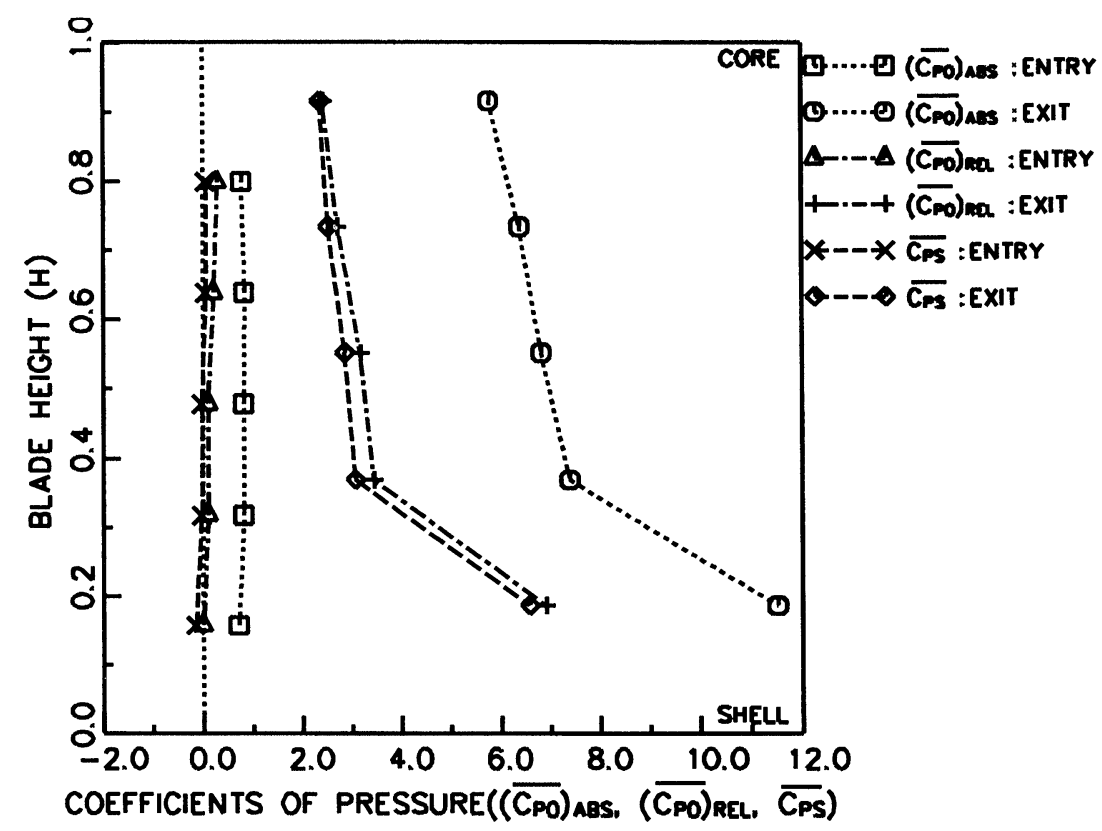

SR 0.8

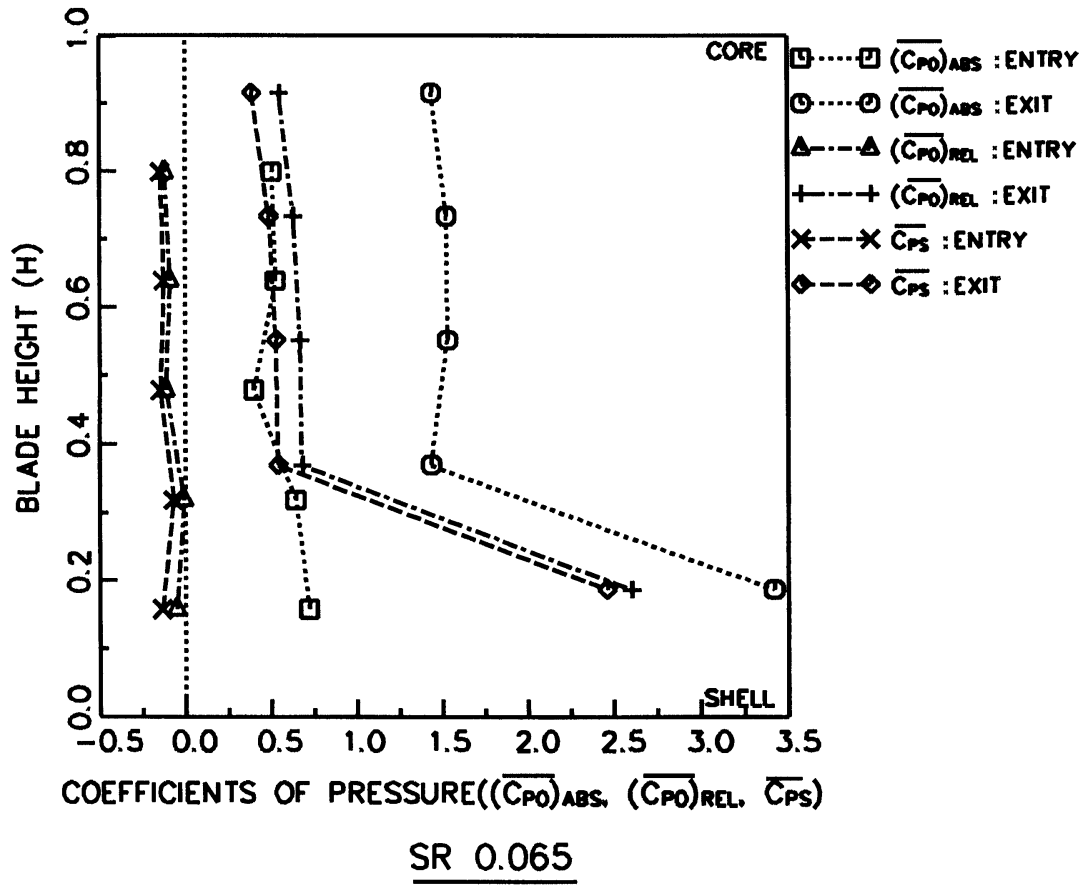

FIGURE 5 Radial distribution of passage-averaged absolute and relative pressure coefficients at SRs 0.8 and 0.065 at pump inlet and exit. 
The relative total velocities are higher at the core than near the mid-span due to the flow overturning at the core, induced by secondary vortex. This phenomenon does not occur at other speed ratios as the secondary flows do not induce flow overturning near the core.

The distribution of static pressure is found to be similar for both speed ratios (Marathe, 1998). However, the magnitudes change significantly for each speed ratio as the magnitudes depend on the pump pressure rise which changes significantly from SR 0.065 to 0.8 . For both speed ratios, the static pressure increases with an increase in the radius. It should be noted that the variation of relative total velocity (presented later) does not affect the distribution of static pressure as the relative dynamic head does not change significantly inside the pump passage. Lower static pressure near the core and higher static pressure near the shell is caused by lower diffusion of velocities near the core and higher diffusion near the shell as explained earlier in the paper. The jump in the radial distribution of $\overline{C_{P S}}$ near shell (Fig. 5) is due to the sudden deceleration of the flow on the turbine shell as explained earlier in the paper.

\section{Velocity Profiles and Angles at the Pump Exit}

The normalized relative total velocity, at SR 0.8 (Fig. 6), shows a large region of flow separation near core suction side. This region extends beyond mid-span and up to $25 \%$ of the passage width near SS. As a result, the high momentum flow is transported towards pressure side-shell region. The distribution of relative total velocity is mainly influenced by the axial velocity. The magnitudes of radial velocity and relative tangential velocity are small at the exit of pump. The radial velocity distribution (Marathe, 1998) shows radial positive velocity in the entire passage except near the pressure side of the wake and in the separation zone. The relative tangential velocity shows flow underturning except inside the separation zone and inside the wake. This can be attributed to the flow mixing downstream of the pump.

The relative total velocity distribution for SR 0.065 (Fig. 6) is significantly different from that observed for SR 0.8 . The relative total velocity distribution shows a thinner diffused wake near the core SS but a large region of low momentum near shell SS. This is attributed to the flow separation at SS shell corner or transportation of separation/ mixing region towards the shell. The transport is caused by strong secondary flow generating a passage vortex which occupies the entire passage. The center of the vortex corresponds to the low relative total velocity. The strong passage vortex also transports the high momentum region from shell pressure side towards the core. Thus the high momentum region extends near the pressure side from shell to core. The axial velocity profile (Marathe, 1998) does not show any region of separation. However, a low velocity zone is observed near the center of vortex. The strong passage vortex also causes outward radial velocities near the suction side and inside the wake and inward radial velocities near the meridional plane. It also induces underturning near the shell and overturning near the core region which is observed in the distribution of the tangential velocity (Marathe, 1998).

The radial distribution of passage averaged velocities (normalized), from shell to core at SR 0.8 show (Fig. 7) higher velocities near the shell and lower velocities near the core. The absolute total velocity is dominated by the absolute tangential velocity whereas the relative total velocity is dominated by the axial velocity. The magnitudes of relative tangential velocity and radial velocity are negligible. As indicated earlier, the axial velocities are low near the core resulting from flow separation inside the passage at this location. All velocity components increase towards the shell due to the blockage caused by this flow separation.

The radial distribution of normalized passage averaged velocities at SR 0.065 shows (Fig. 7) nearly uniform velocities from core to shell. A radial 

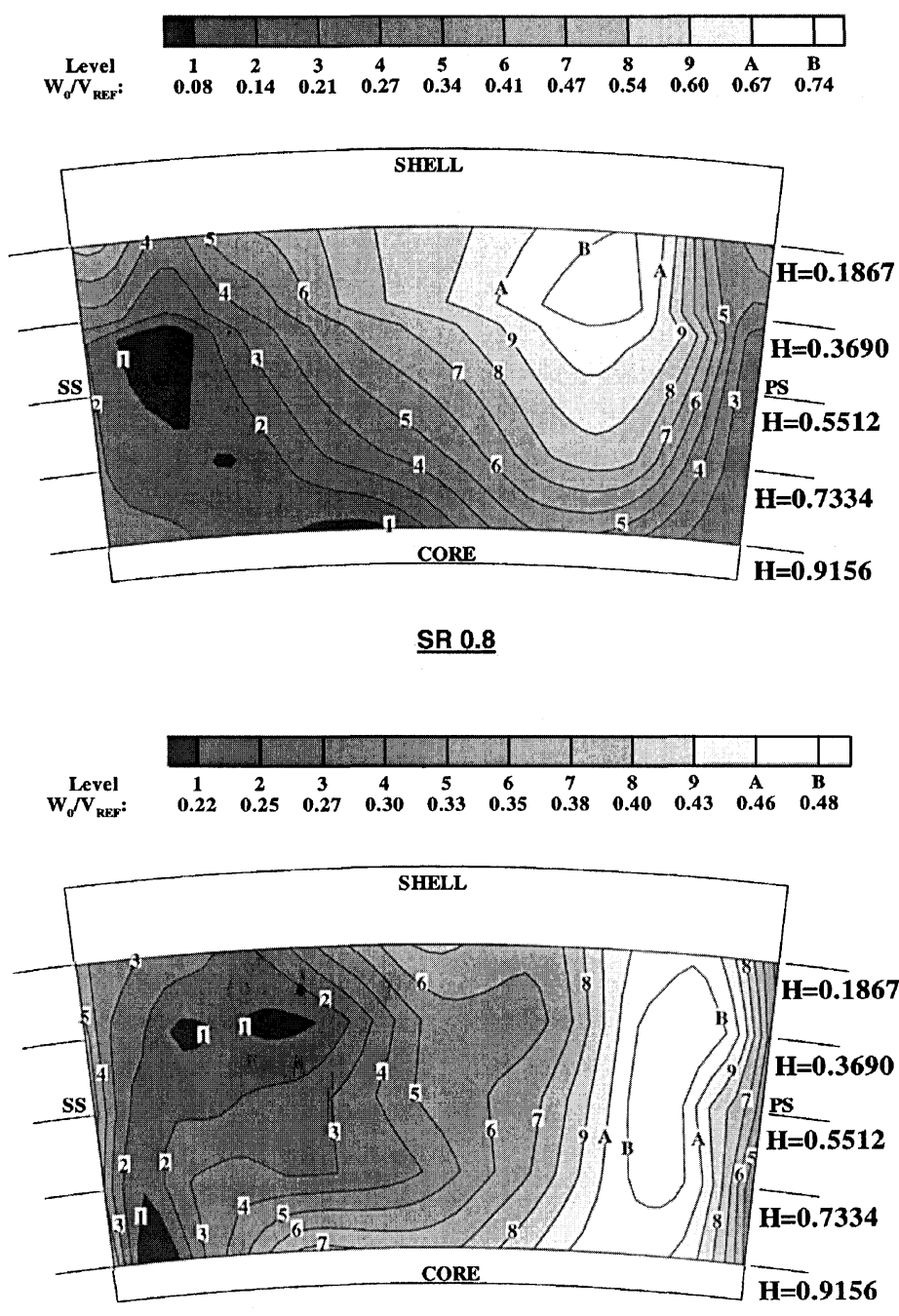

SR 0.065

FIGURE 6 Contours of normalized relative total velocity at SRs 0.8 and 0.065 at pump exit.

redistribution of mass flow takes place inside the pump passage by transport of the high momentum flow near the core. This is caused by a strong secondary vortex as indicated earlier. The flow overturning near the shell is also evident.

The relative yaw angle distribution at SR 0.8 show (Fig. 8) overall flow underturning in most of the passage except near the suction side of wake/ separation zone, due to low and negative axial velocities in the separation region. The overall change in the yaw angle is large (approximately $280^{\circ}$ ). However, in the core flow region the variation is about $30^{\circ}$. This fact is confirmed by the radial distribution of the flow angles (Fig. 9) calculated from the passage-averaged values of velocities, at each radius. The radial variation of pitch angle distribution shows radially positive angles everywhere in the passage except near the core. The overall variation of the pitch angle is $15^{\circ}$ which was also observed from the contour plots (Marathe, 1998).

The flow angle distribution at SR 0.065 (Fig. 8) shows significantly different distribution. High flow overturning near the core and high 

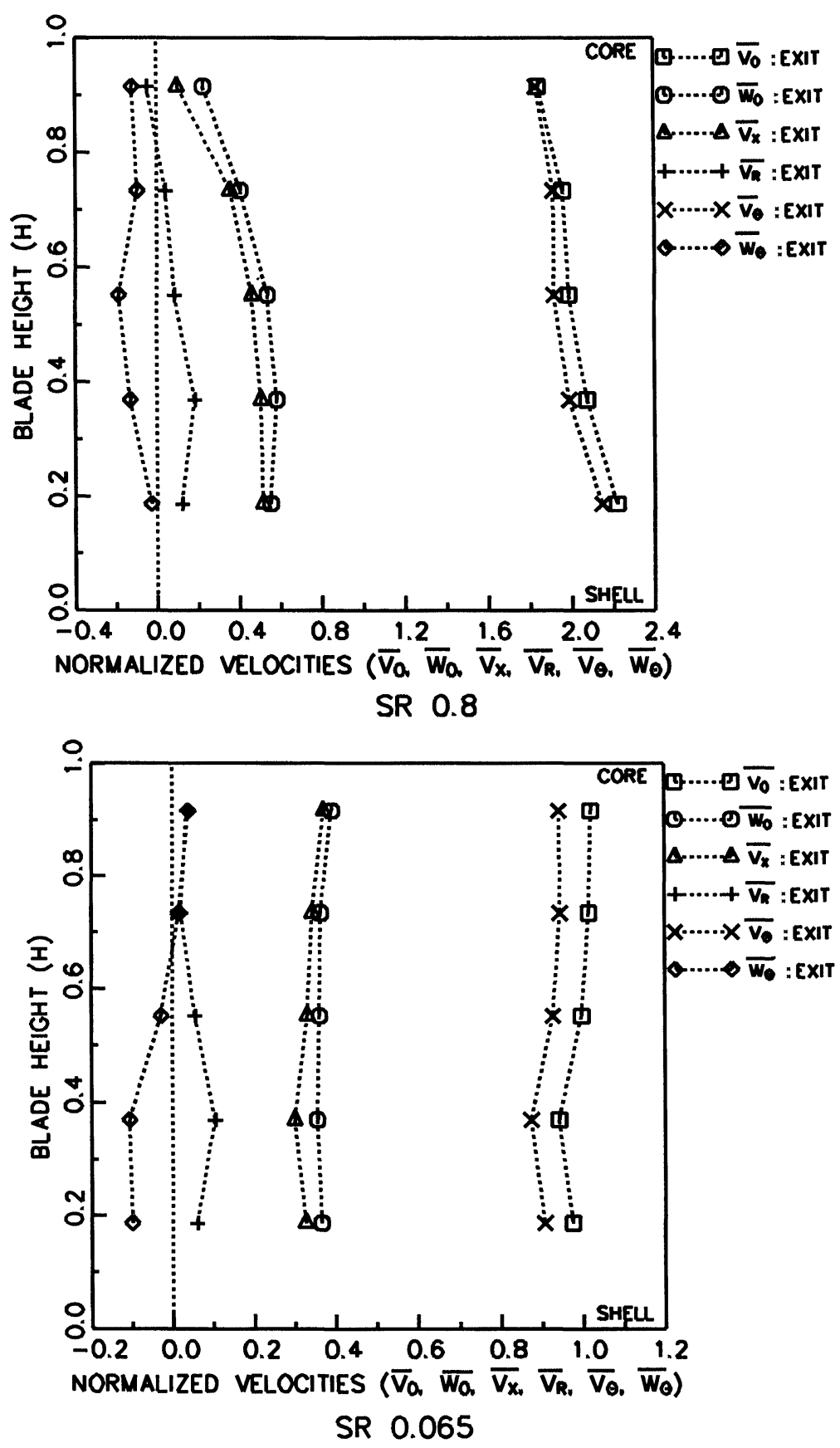

FIGURE 7 Radial distribution of passage-averaged normalized velocity components at SRs 0.8 and 0.065 at pump exit.

flow underturning near the shell is clearly observed. The flow overturning region is located near the suction surface core and occupies nearly half the passage width and height. The radial distribution of the passage averaged flow angles reveals underturning of the flow near the core. The overall variation of yaw angle is about $30^{\circ}$ from core to shell. The radial flow angles are positive throughout the passage (Marathe, 1998). The radial flow angles increase from core to $H=0.3960$ and 


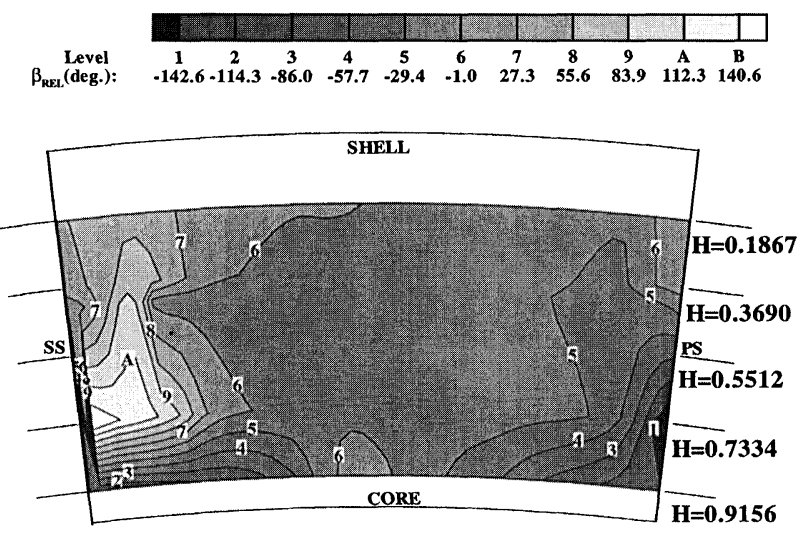

$\underline{\text { SR } 0.8}$

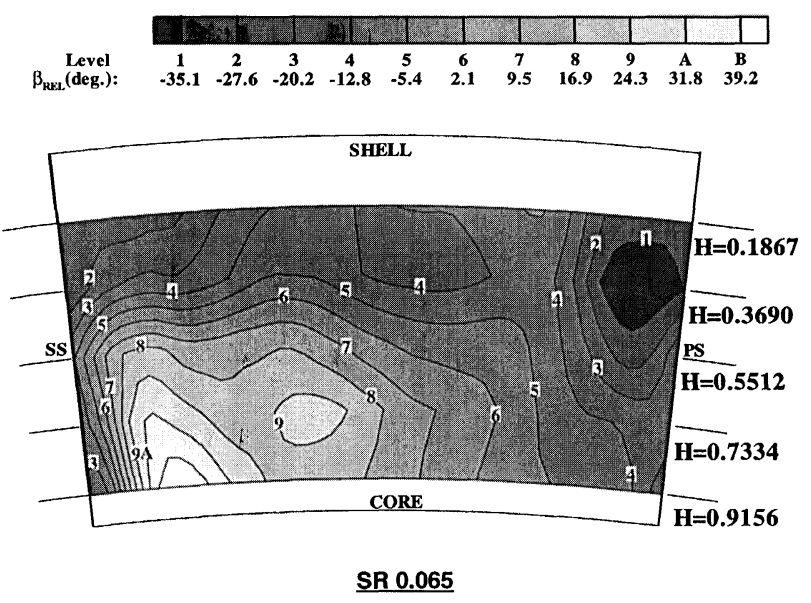

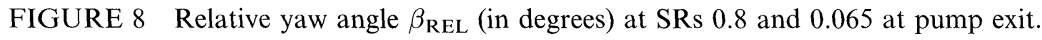

decrease again near the shell. This decrease is due to the change in direction of secondary flow due to the passage vortex.

\section{Secondary Flow Vectors at Pump Exit}

The secondary flow velocity $\mathbf{W}_{\mathrm{SEC}}$ is defined as

$\mathbf{W}_{\mathrm{SEC}}=\mathbf{i}_{\Theta}\left[\left(W_{\Theta}\right)_{\mathrm{MEASURED}}-\left(W_{\Theta}\right)_{\mathrm{IDEAL}}\right]+\mathbf{i}_{R} W_{R}$

where

$$
\left(W_{\Theta}\right)_{\text {IDEAL }}=\left(W_{X}\right)_{\text {IDEAL }} \times \tan \left(\beta_{\text {BLADE-EXIT }),}\right.
$$

and $\beta_{\text {BLADE-EXIT }}$ is defined as the pump exit blade angle.

The secondary flow vectors at SR 0.8 show (Fig. 10) a weak vortex structure near the separation zone. The secondary flow vortex rotates in anti-clockwise direction. This vortex is a result of the turning of the boundary layer, rotation of the boundary layer and rotation of the torus flow as analyzed by Lakshminarayana and Horlock (1973) and explained by Backström and Lakshminarayana (1996) and By et al. (1995). A clockwise vortex can be seen near the pressure side from core to shell. The presence of streamwise vorticity at the inlet of the pump (exit of the stator) cause the secondary flow 


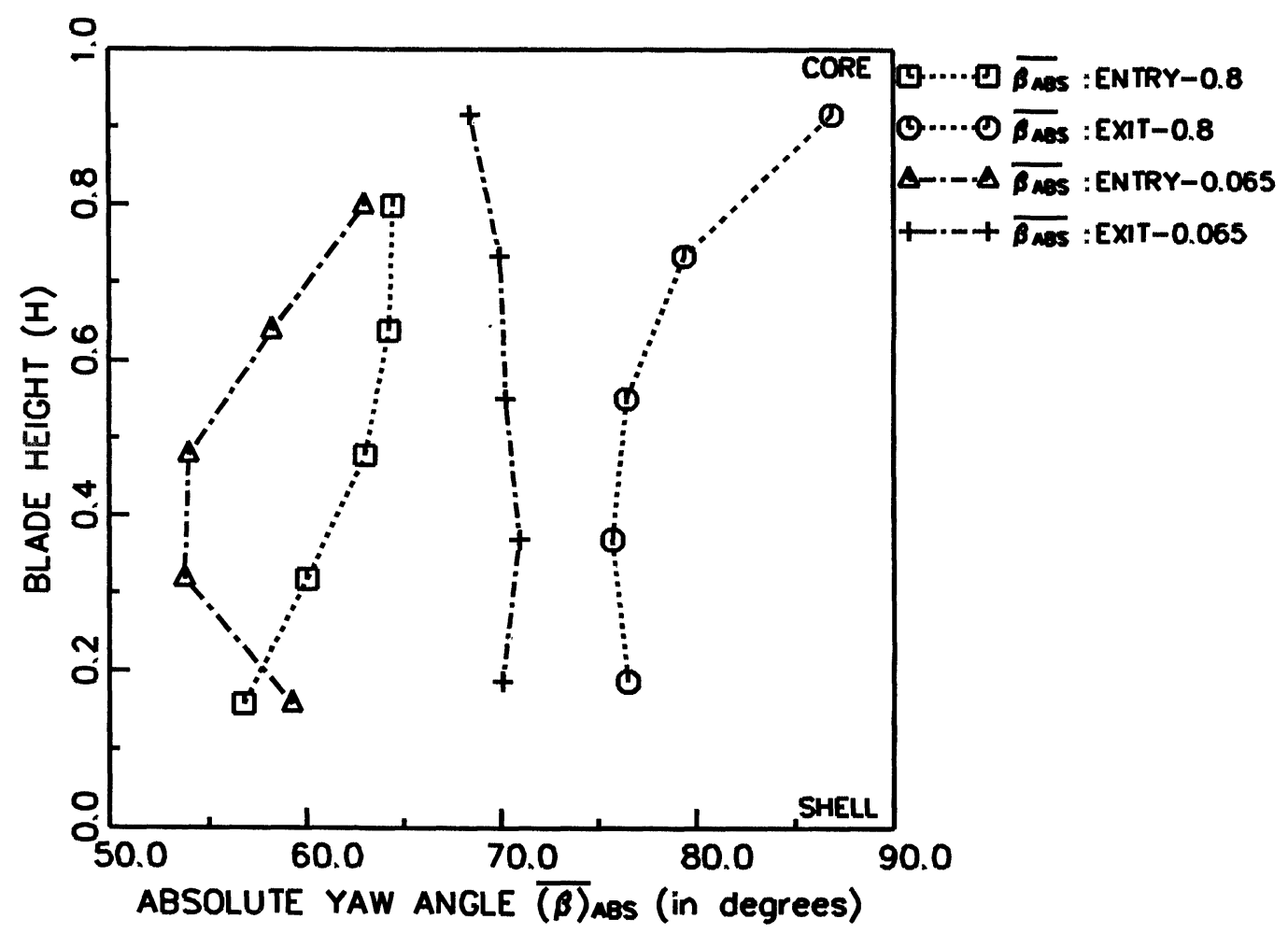

FIGURE 9 Radial distribution of time-averaged absolute flow angles at SRs 0.8 and 0.065 at pump exit.

to deviate considerably from those observed in isolated rotors or cascades.

The secondary flow pattern at SR 0.065 (Fig. 10) is significantly different from that observed for SR 0.8 . A strong passage vortex is observed rotating in clockwise direction. This vortex occupies the entire passage and its center is not in the wake/separation zone. Also, this vortex induces considerable redistribution of the flow at SR 0.065. This passage vortex could be caused by complex interaction of separated flow regions and boundary layers inside the pump passage combined with the rotation effects, as explained by Lakshminarayana (1996).

\section{DISTRIBUTION OF UNRESOLVED UNSTEADINESS DOWNSTREAM OF PUMP}

Radial distribution of the RMS values of each of the unsteady components (periodic, aperiodic and unresolved) downstream of the pump (upstream of the turbine) is presented in this section. The equations for calculation of these quantities are presented by Marathe et al. (1997) and will not be repeated here. All the quantities presented here are in the absolute frame of reference.

The unsteady velocity fluctuations at SR 0.8 show (Fig. 11) that the overall unsteadiness in total and tangential velocities is dominated by unresolved unsteadiness with approximately $40 \%$ contribution from the periodic unsteadiness. The position of the maximum unresolved unsteadiness coincides with the position of the secondary vortex. The unsteady axial and radial velocities show significant contribution from both the periodic and the unresolved unsteadiness. For the axial velocity, the periodic unsteadiness dominates the overall unsteadiness (Marathe, 1998).

The total unsteadiness, which includes the blade periodic (blade-to-blade variation of properties at 


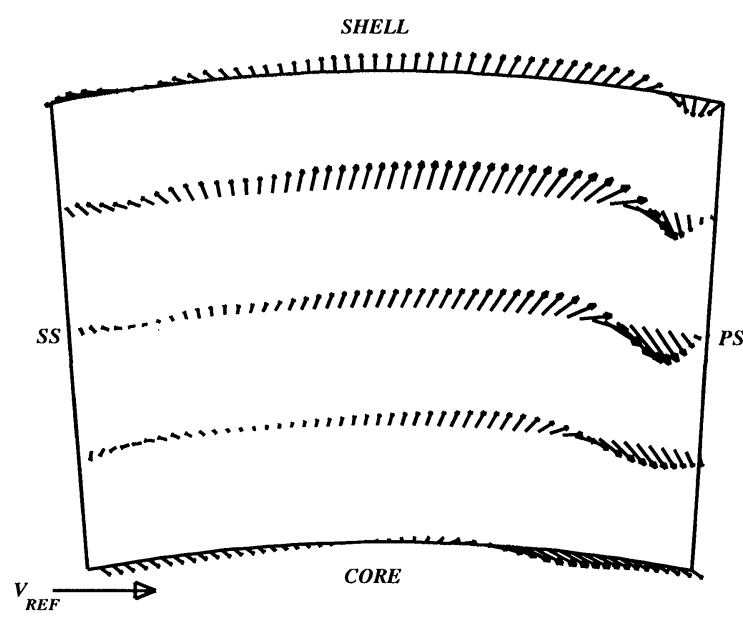

SR 0.8

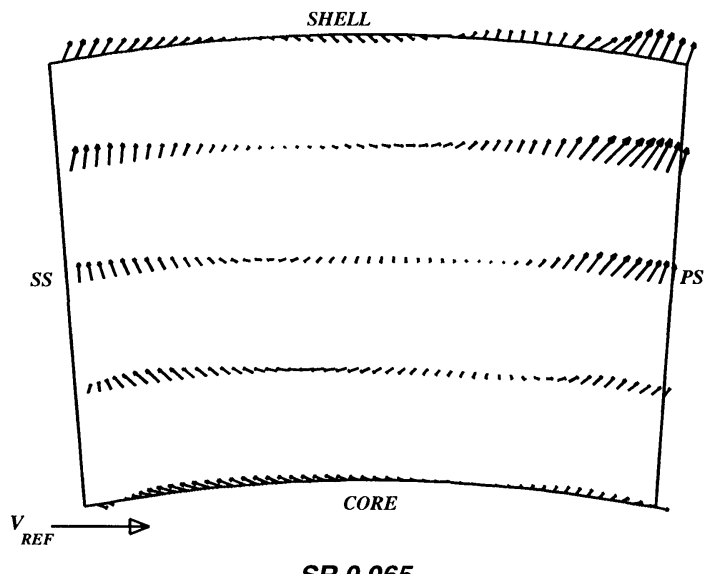

$\underline{\text { SR } 0.065}$

FIGURE 10 Secondary flow vectors at SRs 0.8 and 0.065 at pump exit.

pump exit) and all pump unsteadiness not linked with the pump rotor or pump blade passing frequencies, is very high. The magnitudes are about $25 \%$ of $V_{\mathrm{REF}}$. The aperiodic component due to variation of flow properties from one revolution to other and the stator-rotor interaction or rotorrotor (pump-turbine) interaction is small. The frequency spectrum of the pump exit data (Marathe, 1998) indicate that the influence of downstream turbine is small.

For SR 0.065 (Fig. 11) the blade periodic fluctuations of the velocity play a more significant role due to a strong passage vortex covering the entire passage. The overall unsteadiness is lower near the mid-span and higher near the core due to the flow redistribution at this location.

The radial distribution of RMS values of unsteady yaw angle at both speed ratios (Marathe, 1998) shows approximately $8-10^{\circ}$ of total RMS unsteadiness. For SR 0.8 the unsteadiness increases from approximately $5^{\circ}$ near the shell to about $8-9^{\circ}$ at $H=0.7334$ and decreases to about $7^{\circ}$ near the core. Whereas, for SR 0.065 the total unsteadiness increases from approximately $6-8^{\circ}$ from shell to $H=0.369$ and then remains nearly constant throughout the passage. For both speed ratios the periodic unsteadiness is found to be higher than the unresolved unsteadiness. Such large unsteadiness in flow angle result in off design conditions at the turbine inlet resulting in performance deterioration.

\section{CONCLUDING REMARKS}

The distribution of flow properties at the exit of the pump of an automotive torque converter, in the relative reference frame, shows a very non-uniform flow field across the entire flow passage. The flow near the suction side core is separated for SR 0.8 and nearly separated for SR 0.065 . As a result, the high momentum flow is transported towards the pressure side. The static and total pressure distributions at both speed ratios show a sudden increase near the shell. This could be possibly caused by the significant transport of high momentum flow towards the shell.

A secondary vortex, rotating anti-clockwise, is observed near the suction side for SR 0.8. This secondary vortex interacts with the separation region and wake causing accelerated diffusion in this region and flow overturning near the core. The secondary vortex at SR 0.065 rotates in the clockwise direction and encompasses the entire passage. The unresolved unsteadiness of all properties dominate the unsteady flow field, which is consistent with the data reported by Brun et al. (1996). The unresolved unsteadiness in the velocity at the 


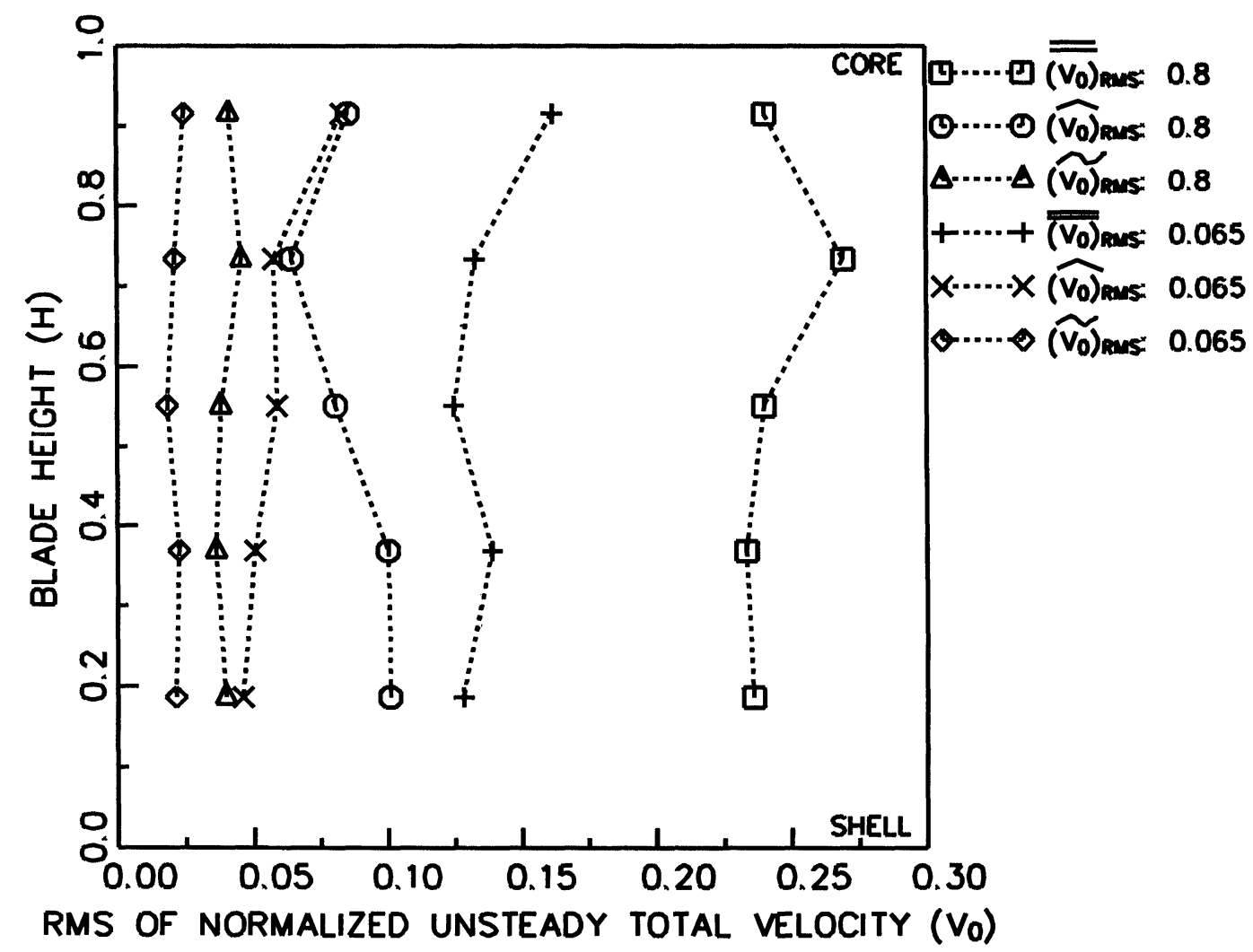

FIGURE 11 Radial distribution of passage-averaged value of RMS of normalized absolute total velocity fluctuations at SRs 0.8 and 0.065 at pump exit.

pump exit is high (approximately $25 \%$ of $V_{\mathrm{REF}}$ and $12 \%$ of the mean value) near the core and moderate near the shell (approximately $15 \%$ of the $V_{\mathrm{REF}}$ and $8 \%$ of mean value). These magnitudes indicate very high levels of turbulence at both the core and the shell regions. The periodic component generated due to the blade-to-blade distribution of the steady state flow properties in the pump reference frame is significant. The aperiodic component due to rotorrotor interaction was not found significant. Although, the fluctuations in radial and axial components of the velocity are as high as tangential velocity, the unsteadiness in the tangential velocity and static pressure show maximum influence on the unsteady total velocity and stagnation pressure. Unresolved unsteadiness is higher in the regions of flow separation and secondary vortex.
The radial distribution of flow properties upstream and downstream of the pump indicates higher losses near the core and lower losses near the mid-span and shell at SR 0.8. High losses are caused by flow separation and secondary vortex generating a low momentum region near the core.

It can be concluded that the flow field inside a torque converter is highly three dimensional, viscous and unsteady. Hence, the effects of three dimensionality and unsteady effects should be incorporated in the design of the torque converter elements to achieve improved performance.

\section{Acknowledgments}

This work was sponsored by Powertrain Division of General Motors Corporation. The authors wish to 
express their gratitude to Mr. Donald G. Maddock for his assistance and comments on the experimental program. Assistance by Mr. Yu Dong in the data acquisition is also acknowledged.

\section{NOMENCLATURE}

\section{$C$}

$\left(C_{P 0}\right)_{\mathrm{REL}} \quad 2\left(\left(P_{0}\right)_{\mathrm{REL}}-P_{\mathrm{HUB}}\right) / \rho V_{\mathrm{REF}}^{2}$

$\left(C_{P 0}\right)_{\mathrm{ABS} .} . \quad 2\left(\left(P_{0}\right)_{\mathrm{ABS}}-P_{\mathrm{HUB}}\right) / \rho V_{\mathrm{REF}}^{2}$

$C_{P \mathrm{~S}} \quad 2\left(P_{\mathrm{S}}-P_{\mathrm{HUB}}\right) / \rho V_{\mathrm{REF}}^{2}$

$H \quad$ Spanwise distance from shell (outer side) normalized by the blade span ( $H=0$ at the shell, 1 at the core)

$P \quad$ Pressure normalized by $\rho V_{\mathrm{REF}}^{2} / 2$

$P_{\text {HUB }} \quad$ Reference pressure on the stator hub

$P S$, SS Pressure and suction side

$S \quad$ Blade spacing

SR $\quad$ Speed ratio (turbine $\mathrm{rpm} / \mathrm{pump} \mathrm{rpm}$ )

$U \quad$ Blade speed

$V \quad$ Absolute velocity normalized by $V_{\text {REF }}$

W Relative velocity normalized by $V_{\text {REF }}$

$V_{\text {REF }} \quad$ Normalizing velocity $\left(U_{\text {TIP PUMP }} \sqrt{1-\mathrm{SR}}\right)$

$\alpha \quad$ Pitch angle, $\tan ^{-1}\left(V_{R} / V_{X}\right)$, positive in the radial outward direction

( ) $)_{\text {RMS }} \quad$ The root mean square values of fluctuating quantities $\left(V, P_{0}, P_{\mathrm{S}}, \alpha, \beta\right)$

()$_{\text {RMS }} \quad$ Blade periodic RMS values of fluctuating quantities

\section{Subscripts}

1,2 Inlet and exit of the pump

0, S Total/stagnation, static

ABS Absolute (stator) reference frame
REL Relative (pump) reference frame

$X, R, \Theta \quad$ Axial, radial and tangential components

hub, tip Property at the hub and tip, respectively

RMS Root mean square value

\section{Superscripts}

$\begin{array}{ll} & \text { Time-averaged value (Eq. (2), } \\ \text { Marathe } \text { et al., 1997) } \\ \text { Blade periodic component (Eq. (5), } \\ \text { Marathe } \text { et al., 1997) } \\ \text { Blade aperiodic component (Eq. (6), } \\ \text { Marathe } \text { et al., 1997) } \\ \text { Unresolved component (Eq. (7), } \\ \quad \text { Marathe } \text { et al., 1997) } \\ \quad \text { Passage-averaged value }\end{array}$

\section{References}

Backström, T.W. Von and Lakshminarayana, B. (1996) Fluid dynamics and performance of automotive torque converters: an assessment, Journal of Fluids Engineering, 118, 665-676.

Browarzik, V. and Grahl, K.G. (1992) Non-steady flow measurements inside a hydrodynamic torque converter by hot-film anemometry, ASME Paper 92-GT 161.

Brun, K., Flack, R.D. and Gruver, J.K. (1996) Laser velocimeter measurements in the pump of a torque converter part II - unsteady measurements, Journal of Turbomachinery, $118(3), 570$.

By, R.R., Kunz, R.F. and Lakshminarayana, B. (1995) NavierStokes analysis of the pump flow field of an automotive torque converter, Journal of Fluids Engineering, 117(1), 116-122.

Gruver, J.K., Flack, R.D. and Brun, K. (1996) Laser velocimeter measurements in the pump of a torque converter part I average measurements, Journal of Turbomachinery, 118(3), $562-569$.

Lakshminarayana, B. (1996) Fluid Dynamics and Heat Transfer of Turbomachinery, John Wiley \& Sons Inc, New York.

Lakshminarayana, B. and Horlock, J.H. (1973) Generalized expressions for secondary vorticity using intrinsic coordinates, Journal of Fluid Mechanics, 59, 97-115.

Marathe, B.V. (1998) Investigation of three-dimensional, unsteady, viscous flow field inside an automotive torque converter, Ph.D. Thesis, The Pennsylvania State University.

Marathe, B.V., Lakshminarayana, B. and Maddock, D.G. (1997) Investigation of steady and unsteady flow field downstream of an automotive torque converter and inside the stator, part-I and II, Journal of Turbomachinery, 119, 624-645. 


\section{ait \\ ENERGY MATERIALS}

M A N E Y publishing

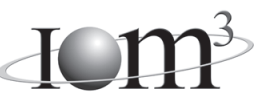

\section{Materials Science \& Engineering for Energy Systems}

Maney Publishing on behalf of the Institute of Materials, Minerals and Mining

The Institute of Materials, Minerals \& Mining

Economic and environmental factors are creating ever greater pressures for the efficient generation, transmission and use of energy. Materials developments are crucial to progress in all these areas: to innovation in design; to extending lifetime and maintenance intervals; and to successful operation in more demanding environments. Drawing together the broad community with interests in these areas, Energy Materials addresses materials needs in future energy generation, transmission, utilisation, conservation and storage. The journal covers thermal generation and gas turbines; renewable power (wind, wave, tidal, hydro, solar and geothermal); fuel cells (low and high temperature); materials issues relevant to biomass and biotechnology; nuclear power generation (fission and fusion); hydrogen generation and storage in the context of the 'hydrogen economy'; and the transmission and storage of the energy produced.

As well as publishing high-quality peer-reviewed research, Energy Materials promotes discussion of issues common to all sectors, through commissioned reviews and commentaries. The journal includes coverage of energy economics and policy, and broader social issues, since the political and legislative context influence research and investment decisions.

\section{CALL FOR PAPERS}

Contributions to the journal should be submitted online at http://ema.edmgr.com

To view the Notes for Contributors please visit: www.maney.co.uk/journals/notes/ema

Upon publication in 2006, this journal will be available via the Ingenta Connect journals service. To view free sample content online visit: www.ingentaconnect.com/content/maney

For further information please contact:

Maney Publishing UK

Tel: +44 (0)113 2497481 Fax: +44 (0)1132486983 Email: subscriptions@maney.co.uk

or

Maney Publishing North America

Tel (toll free): 8662975154 Fax: 6173546875 Email: maney@maneyusa.com

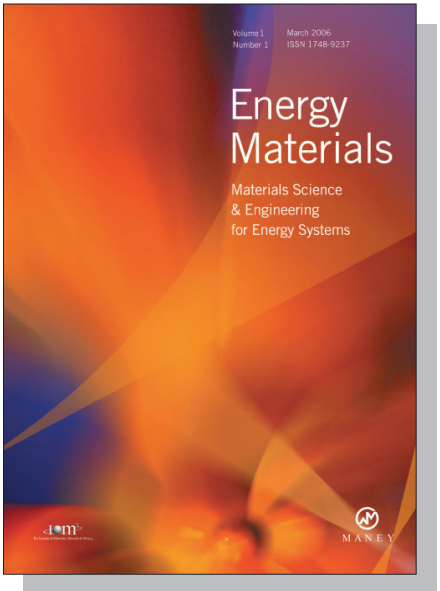

EDITORS

Dr Fujio Abe

NIMS, Japan

Dr John Hald, IPL-MPT, Technical University of Denmark, Denmark

Dr R Viswanathan, EPRI, USA

\section{SUBSCRIPTION INFORMATION}

Volume 1 (2006), 4 issues per year

Print ISSN: 1748-9237 Online ISSN: 1748-9245

Individual rate: $£ 76.00 / U S \$ 141.00$

Institutional rate: $£ 235.00 /$ US $\$ 435.00$

Online-only institutional rate: $£ 199.00 / U S \$ 367.00$

For special $\mathrm{IOM}^{3}$ member rates please email

subscriptions@maney.co.uk

\section{For further information or to subscribe online please visit www.maney.co.uk}



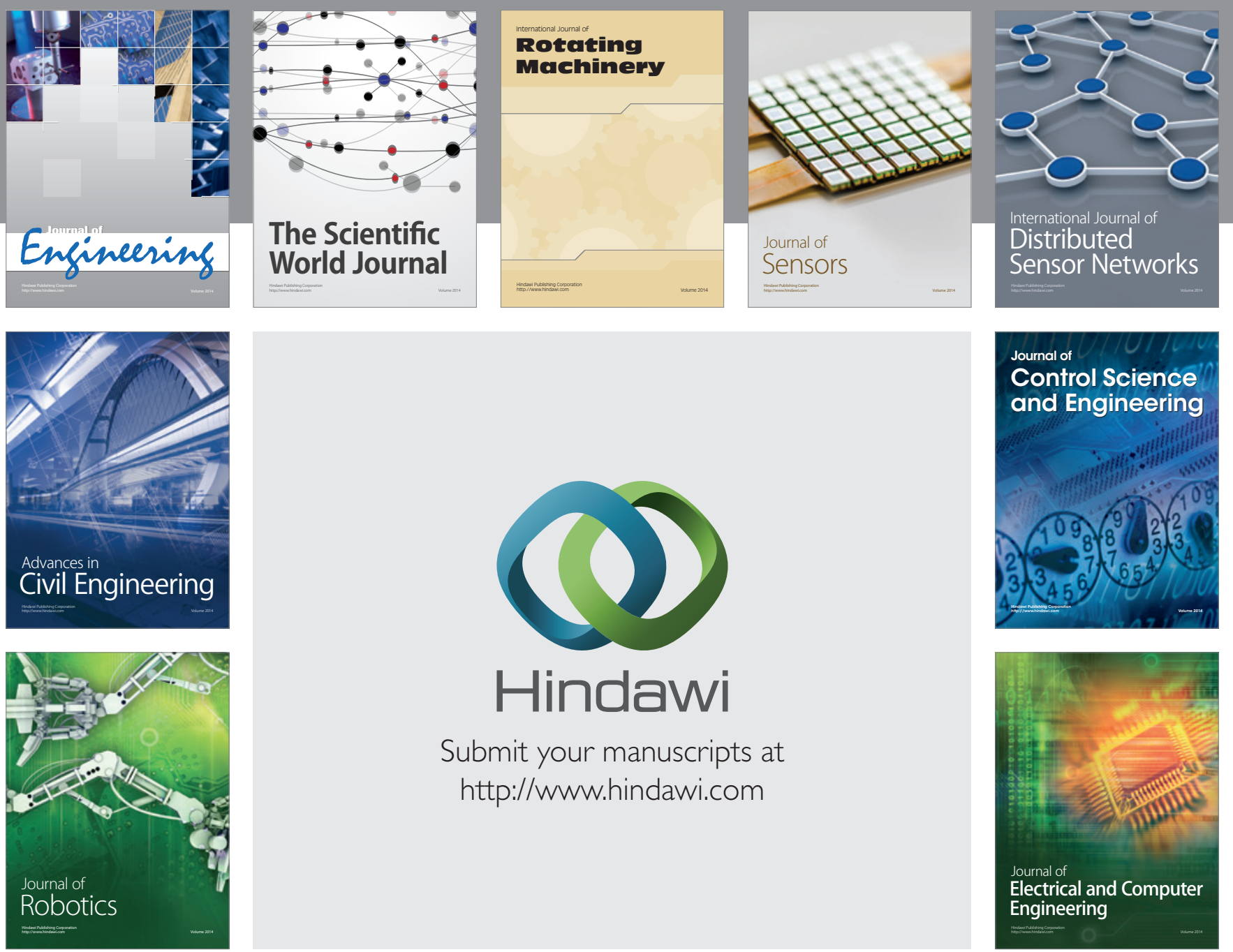

Submit your manuscripts at

http://www.hindawi.com
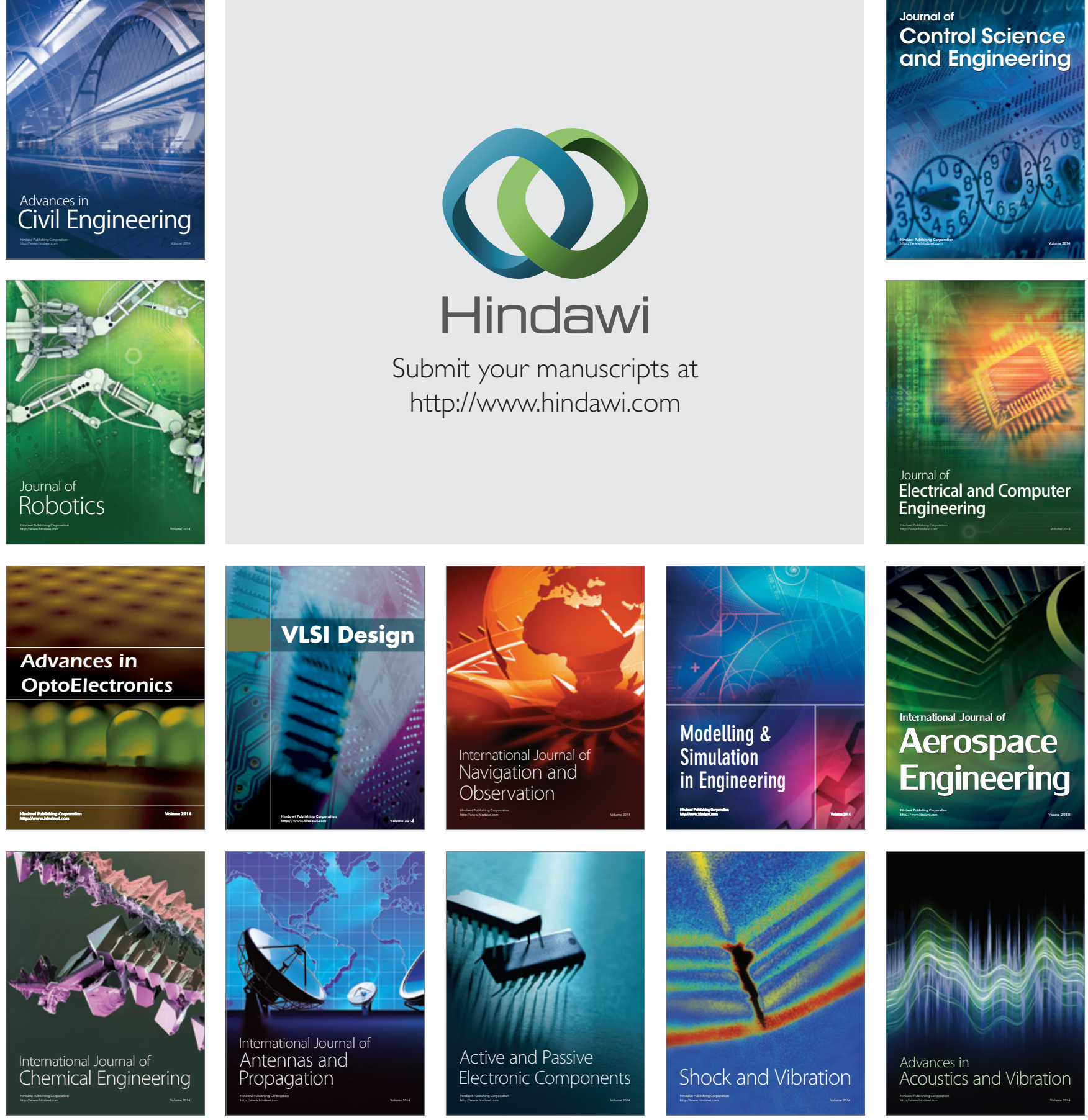